\title{
A film video processor for the viewing of fluorescein angiograms
}

\author{
MICHAEL STUR \\ From the Second Department of Ophthalmology, University of Vienna, Austria
}

SUMMARY A new, commercially available, and inexpensive film video processor facilitates the viewing of fluorescein angiograms on video monitors. The negative film is inserted into a film carrier, read by a charge-coupled device solid state pickup device and presented as a negative or positive image on a black-and-white monitor with the help of a negative/positive conversion feature. Details of the angiogram are studied by zooming into any part of the image, which magnifies the image up to six times. Contrast and brightness adjustment on the video monitor allows examination of under- or overexposed angiograms.

Fluorescein angiograms are usually examined with the help of loupes, microfilm readers, or slide projectors. ${ }^{2}$ Despite the fact that the negative film provides more details, many ophthalmologists prefer to study positive images of the angiogram obtained by contact printing on a sheet of transparent film, a procedure which takes time and increases the expense considerably. The presentation of positive images of angiograms on video screens would reduce considerably the cost of production of the positive image as well as the time until the angiogram can be

Correspondence to Dr Michael Stur, 2 Augenklinik, Alserstrasse 4, A-1090 Vienna, Austria. evaluated. A custom-made closed-circuit television system, which can also be used for the presentation of fluorescein angiograms, was presented recently. ${ }^{3} \mathrm{We}$ tested a new, commercially available, and inexpensive film video processor in order to evaluate its qualification for the presentation of fluorescein angiograms.

\section{Material and methods}

The Tamron Fotovix film video processor (Tamron Co., Omiya Saitama, Japan) is a small, compact (width $\times$ height $\times$ depth: $190 \times 230 \times 350 \mathrm{~mm}$ ) and light

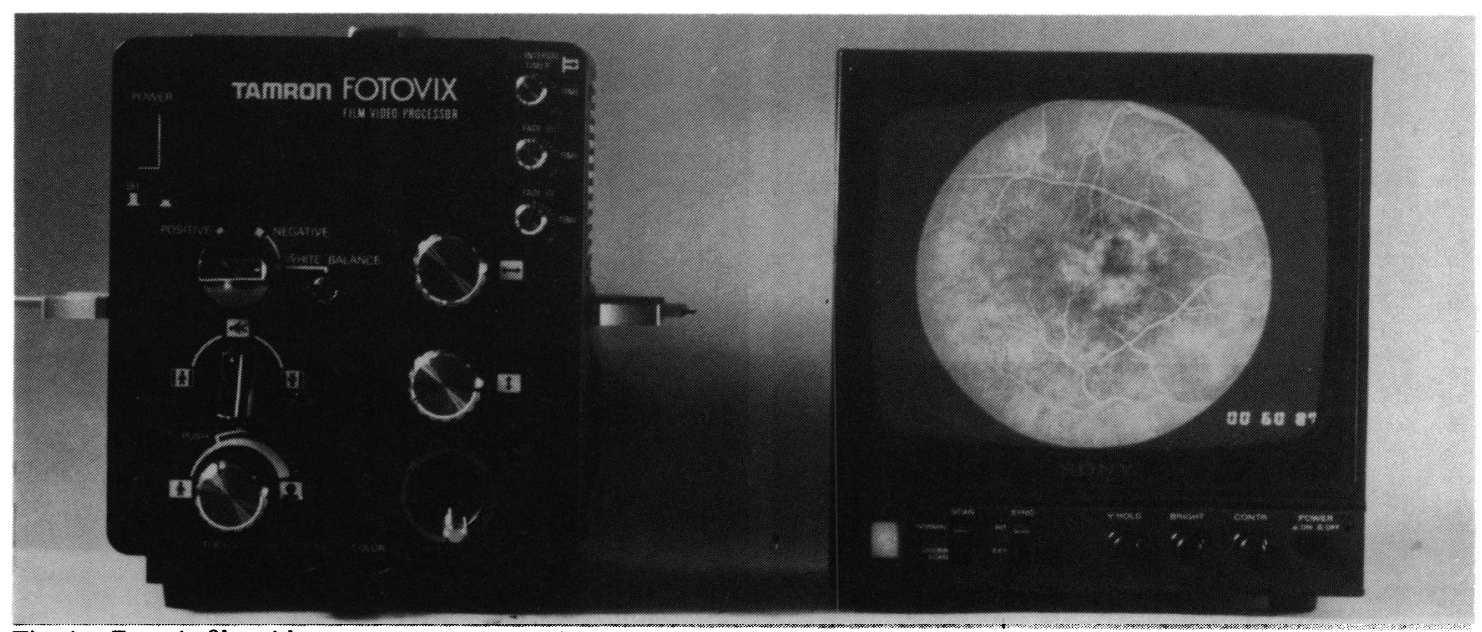

Fig. 1 Fotovix film video processor connected to a 9-inch $(22.9 \mathrm{~cm})$ black-and-white monitor. 


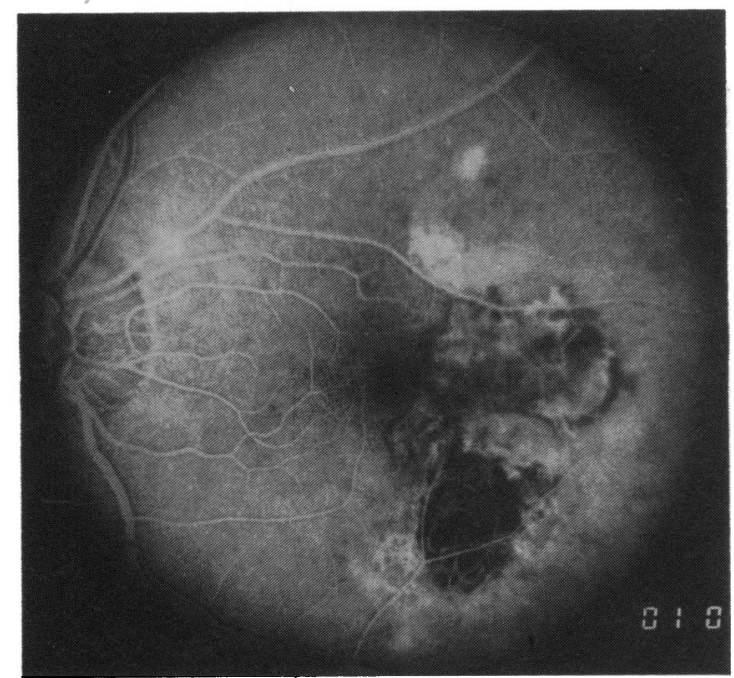

Fig. 2 Conventional print of a fluorescein angiogram showing a parafoveal subretinal neovascular membrane adjacent to a chorioretinal scar caused by ocular toxoplasmosis. 


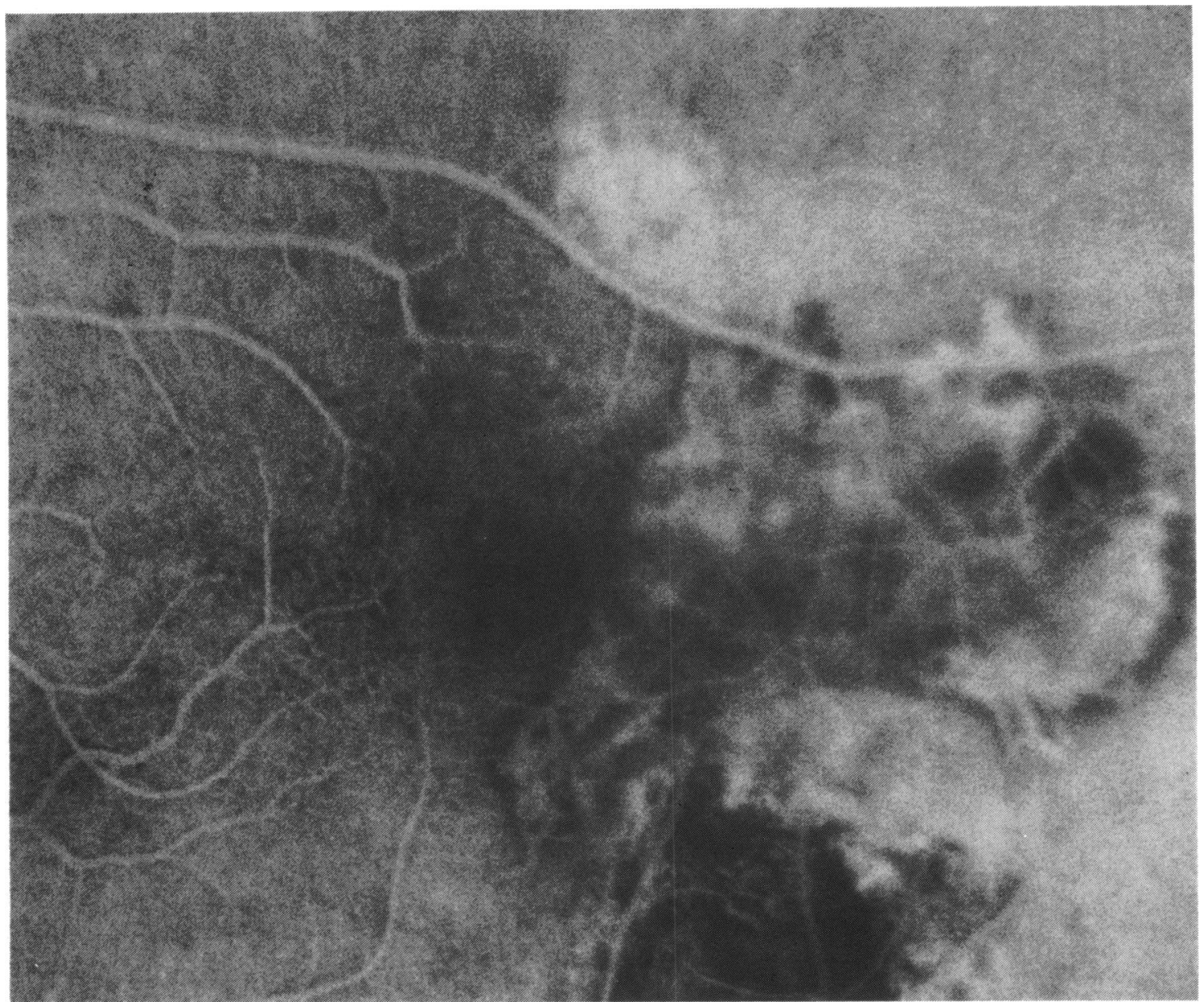

Fig. 5 Conventional print with the same magnification as Fig. 4. Owing to the limits of the film resolution the difference from Fig. 4 is not significant.

weight $(6 \mathrm{~kg})$ unit which contains a stage for $35 \mathrm{~mm}$ film or slide carriers, a charge-coupled device (CCD) solid state colour pickup device with a zoom lens, an illumination system, a negative/positive conversion feature, and controls for horizontal and vertical adjustment, focus, brightness, and colour (Fig. 1). The film carrier is large enough to hold a $35 \mathrm{~mm}$ negative film strip with six frames. Two video output pinjacks, one for colour and one for monochrome, and a radio frequency adapter output are available. We connected a Sony PVM-91CE black-and-white monitor (Sony Co., Japan) to the monochrome video output.

\section{Results}

The quality of the video image was sufficient for evaluation of fluorescein angiograms. When the full frame is presented, small details are not as clearly visible as on a conventional positive print (Figs. 2, 3). Nevertheless the shortcomings of the low resolution of the CCD pickup device might be compensated for with the help of the zooming feature (Fig. 4). The adjustment of the magnification of the image permits a minute examination of the angiogram, and the viewing of several sequential frames is assisted by the horizontal positioning control. Under- or overexposed angiograms may also be examined without difficulty, as the Fotovix performs an automated adjustment of image brightness, which can be finetuned with the brightness and contrast controls of the video monitor.

\section{Discussion}

The evaluation of fluorescein angiograms has gained 
importance because of the increasing frequency of laser treatment for macular diseases. The treatment of a subretinal neovascular membrane requires a recent angiogram, which should be compared with the biomicroscopic findings during the laser treatment procedure. ${ }^{2}$ A single frame of negative or positive film, however, may be inadequate to show subtle details, which are often visible only after comparison of sequential frames. Thus the Fotovix film video processor, which presents the negative or positive image of sequential frames of an angiogram at a preselected magnification, may be able to replace conventional presentation devices even in spite of its shortcomings. Other advantages of the film video processor are the low cost of operation, which might pay off the cost of purchase (price: US\$2198) within a few months, and the possibility of using it for demonstration and teaching. The combination of conventional fluorescein angiography with film video processing and presentation of the angiogram on a video screen is also less expensive than video angiography, which still cannot provide the high resolution of modern 400 ASA black-and-white negative film.

The main disadvantages of the film video processor are the absence of the possibility of a stereoscopic examination and the low resolution in full-frame mode, which prevents the use of the video signal for digital image processing. These disadvantages, however, are of minor importance, since the negative film is still available for stereoscopic examination, printing, or scanning into an image processing system if necessary. Thus the film video processor Fotovix may be recommended for the examination of fluorescein angiograms during laser treatment of macular diseases and for the presentation of angiograms to a larger audience.

This study was supported in part by the Austrian Fund for the Propagation of Scientific Research (Österreichischer Fonds zur Förderung der wissenschaftlichen Forschung, P 5789).

The author has no direct or indirect proprietary interest in this device.

\section{References}

1 Schatz H. Essential fluorescein angiography. San Anselmo: Pacific Medical Press, 1983: 103-24.

2 Patz A, Finkelstein D, Fine St F, Murphy RP. The role of fluorescein angiography in national collaborative studies. Ophthalmology 1986; 93: 1466-70.

3 Folberg R, Verdick RE. Multipurpose closed-circuit television teaching cart. Ophthalmology 1987; 94(S): 19-21.

Accepted for publication 3 November 1988. 